\title{
Usefulness of measuring isokinetic torque and balance ability for exercise rehabilitation
}

\author{
Yong-Seok Jee
}

The goal of exercise rehabilitation is to return to the conditions necessary to be able to engage in specific sports or general physical activity. This type of rehabilitation may be called 'functional treatment'. Functional treatment is symptom guided, injury specific, and sports specific. Recently, many exercise scientists emphasize that functional treatment should be initiated as soon as possible following injury, immobilization or surgery. It hastens the safe return to play any sports and minimizes deconditioning and the harmful effects of immobilization (Buschbacher and Braddom, 1994). A long time ago, many clinicians assembled the specific components of functional treatment noting that each component represents one of the 5 steps in the 'Pyramid of Recovery'. Steps 1 through 5 involved a combined process to gain flexibility and strength. Steps 2 and 3 restored proprioception and endurance in an overlapping manner. After Step 3, the athletes progressed to sport-specific motor relearning and returned to participation based on scientific testing related to sports injuries.

The accurate scientific testing of injured or treated body parts has been the aim of exercise rehabilitation for many years. Exercise scientists who are interested in comparing the effects of training programs seek to precisely measure neuromuscular function and to document the efficacy of exercise rehabilitation in helping injured athletes or patients recovering from injury to the musculoskeletal system regain their muscular conditioning. They have particularly emphasized injury prevention by identifying the bilateral ratio of both limbs and by identifying the ipsilateral ratio between agonist and antagonist muscles of a limb.

Isokinetic dynamometers may be used as scientific devices for testing, comparing, and confirming injured or treated body parts.
Indeed, the constant technological advances over the past $15 \mathrm{yr}$ facilitated the development of highly effective dynamometers usable for the assessment and rehabilitation of most joints in the upper and lower limbs. These devices are regularly used in osteoarticular disorders or in the framework of sports training programs. Most of the isokinetic machines used in pathology are rotating dynamometers. Results of an isokinetic assessment are generally expressed as the peak torque value developed by the tested muscle group. It is also possible to evaluate the work provided by the same muscle group and the power developed at the joint.

After an isokinetic assessment, the isokinetic training protocols are generally described in terms of the modes of muscle concentric or eccentric contraction, value of imposed angular velocity, and number of repetitions. The choice of the training protocol depends on the subject and on the required objectives, as well as for the upper and lower limbs (Hammami et al., 2012). Isokinetic devices allow individuals to exert as much force and angular movement as they can generate to a predetermined velocity. When a limb's angular rate of movement equals or exceeds the preset velocity limit, the dynamometer produces an equaling counterforce to ensure a constant movement rate. Isokinetic resistance has several advantages over other exercise modalities. One advantage is that a muscle group may be exercised to its maximum potential throughout a joint's entire range of motion. It may also provide a safer alternative to other exercise modalities during the process of rehabilitation. It is inherently safer than isotonic training because the dynamometer's resistance mechanism essentially disengages when pain or discomfort is experienced by the patient. In particular, isokinetic training may be used to quan-

This is an Open Access article distributed under the terms of the Creative Commons Attribution Non-Commercial License (http://creativecommons.org/licenses/by-nc/3.0/) which permits unrestricted non-commercial use, distribution, and reproduction in any medium, provided the original work is properly cited. 
tify a muscle group's ability to generate torque or force, and it is also useful as an exercise modality in the restoration of a muscle group's pre-injury level of strength. Isokinetic velocities are usually proposed for the concentric mode range from $0 \% \mathrm{sec}$ to $300 \%$ sec. Distinctions are made between low speeds (from $0 \% \mathrm{sec}$ to $100 \%$ sec), medium speeds (from $100^{\circ} / \mathrm{sec}$ to $200^{\circ} / \mathrm{sec}$ ) and high speeds (from $200 \% \mathrm{sec}$ to $300 \% \mathrm{sec}$ ). The velocities suggested for the eccentric mode are generally lower, never exceeding $150^{\circ} / \mathrm{sec}$. In the number of repetitions, the number for low speeds does not exceed 5 repetitions due to the fatigue induced in trained muscles. For higher speeds, the number of repetitions varies from 5 to 10 repetitions. Endurance tests are frequently carried out at high speed $\left(180^{\circ} / \mathrm{sec}\right.$ minimum) and the number of repetitions may reach 50 repetitions (Rochcongar, 2004).

Balance may be affected by the muscle mass and difference in tolerance (Lee and Hwang, 1984). Many researchers have reported that muscle weakness is associated with an increased risk for falls (Wolfson et al., 1995), hip fractures (Langlois et al., 1998), and adverse physiological changes such as osteoporosis (Sinaki et al., 1986). In order to constantly maintain the body's balance, the center of gravity should be located around the center of the body's cross section. For this reason, the body should maintain the symmetrical positioning and harmoniously contract the flexors and extensors to optimize the movements of the joints. Maintenance of body balance is possible when the postural stability, adequate muscular strength, sensitive balance senses, and accurate reflex functions interact with each other (Lee and Hwang, 1984). According to Panosian and Paige (1995), the body uses the postural and righting reflexes to maintain balance and the vestibular reflex in order to control the muscles of the head, neck, and limbs to stand upright against gravity. The balance ability developed with multiple mechanisms is a vital function in improving the performance of athletics and should be properly maintained during sports activities (Johnson and Nelson, 1986).

In conclusion, the isokinetic test and balance ability test are recommended to injured athletes or those who have undergone operation because those tests can provide accurate scientific results. Moreover, the results from those tests are useful for setting the exercise rehabilitation protocol. Accommodating isokinetic strength with true muscle force or torque curves and accommo- dating balance capacity with precise cognition of the several positions, promises a new and fruitful approach to neuromuscular analysis and rehabilitation training. In other words, the isokinetic device and balance tool can offer many new and exciting applications in the study of understanding human kinesiology.

\section{REFERENCES}

Buschbacher RM, Braddom RL. Sports medicine and rehabilitation: a sport-specific approach. Boston, Mosby, 1994;ix.

Hammami N, Coroian FO, Julia M, Amri M, Mottet D, Hérisson C, Laffont I. Isokinetic muscle strengthening after acquired cerebral damage: a literature review. Ann Phys Rehabil Med 2012;55:279-291.

Johnson BL, Nelson JK. Practical measurements for evaluation in physical education. 4nd ed. Macmillan Publishing Co. 1986;236-239.

Langlois JA, Visser M, Davidovic LS, Maggi S, Li G, Harris TB. Hip fracture risk in older white men is associated with change in body weight from age 50 years to old age. Arch Intern Med 1998;158:990-996.

Lee KH, Hwang KS. Analysis of physical, psychological, and physiological stability on the drawing-release process in archery. Sports Science Institute of Korean Olympic Committee 1984;41-150.

Panosian MS, Paige GD. Nystagmus and postural instability after headshake in patients with vestibular dysfunction. Otolaryngol Head Neck Surg 1995;112:399-404.

Rochcongar P. Isokinetic thigh muscle strength in sports: a review. Ann Readapt Med Phys 2004;47:274-281.

Sinaki M, McPhee MC, Hodgson SF, Merritt JM, Offord KP. Relationship between bone mineral density of spine and strength of back extensors in healthy postmenopausal women. Mayo Clin Proc 1986;61:116-122.

Wolfson L, Judge J, Whipple R, King M. Strength is a major factor in balance, gait, and the occurrence of falls. J Gerontol A Biol Sci Med Sci 1995;50:64-67.

Associate Editor Department of Exercise Physiology \& Prescription, Graduate School of Health Promotion, Hanseo University, 46 Hanseo 1-ro, Haemi-myeon, Seosan 356-706,

Korea E-mail: jeeys@hanseo.ac.kr 